\title{
The Dynamic Review Journal: a scholarly archive
}

\author{
Gary Wills $\dagger \dagger$, Timothy Miles-Board $\dagger$, Christopher Bailey§, Leslie Carr $\dagger$, Quintin \\ Gee§, Wendy Hall $\dagger$ and Simon Grange \\ $\dagger$ Intelligence, Agents, Multimedia Group, University of Southampton, UK \\ $\S$ Learning Technologies Group, University of Southampton, UK \\ qRoyal College of Surgeons of England, London, UK
}

\begin{abstract}
A digital archive, together with its users and its contents, does not exist in isolation - there is a cycle of activity which provides the context for the archive's existence, and which the archive supports through its various roles of information access, discovery, storage, dissemination and preservation. This paper describes an extended digital library environment that we have developed for orthopaedic surgeons which assists in collating and analysing patient data, organising internal project discussions, and producing articles. By bridging the gap between the undertaking of experimental work (surgical trials) and the dissemination of its results through electronic publication, this work addresses the cycle of activity in which a digital archive rests.
\end{abstract}

\section{Introduction}

The accepted role of scientific and scholarly publication is to record research activity in a timely fashion, keeping others in the research community upto-date with current developments. Until very recently, it has been the case that printed journals were the most efficient method for the dissemination and archival of research results. Technical advances in the past decade, however, have allowed the process of scholarly communication to take other forms, particularly in the dissemination and storage of articles via the World Wide Web. Giving publishers a new medium for making their journal archives available (Hitchcock et al. 1996), it also gave authors the means to break the so-called 'Faustian bargain' and directly distribute their articles in pre- or post-publication form from their own Web pages (Harnad 1995) or in organised 'e-print archives' (Ginsparg 1996).

However, it may not be simply the technical support for reproducing and distributing articles electronically (electronic publishing, e-print archiving and digital libraries), but also the emergence of technical support for improving human communication in the form of highly collaborative, large-scale activities

‡ Corresponding author. Email: gbw@ecs.soton.ac.uk 
and analyses (the Grid, Virtual Universities) that is likely to precipitate significant change in the field of scientific communication and significant changes in the way its communications are produced, curated and disseminated (Lucier 1990). For example, the old medium allowed a paper to be published as the summarisation of a scientific activity - the raw observations that led to the article's conclusions are replaced by a description of the method for recreating the experiment. However, researchers are becoming more interested in the potential of the new medium for preserving experimental data and methodology as well as experimental conclusions (Frey et al. 2002): the ability to provide hypertext links between the article and the data to create an 'audit trail' for reviewers and thus facilitate further analyses and meta-analyses.

Our work within the Virtual European Orthopaedic University (VOEU) project has enabled us to contribute to this broadening of the traditional view of digital archives as merely collections towards the processes of collecting and deploying. We have already discussed the role of digital library systems in the undertaking of science in detail in our previous paper (Carr et al. 2004); the purpose of this paper is to focus more on our development of an extended digital library environment for orthopaedic learning, training and research. We discuss how existing orthopaedic research practice motivated the development of a computer-based support environment (Section 3), describe the architecture of the environment itself (Section 4) and our approach to evolving it to the changing needs of its users (Section 5). We then discuss how the DRJ could be applied to other online scientific communities (Section 6), provide an account of the system in use (Section 7), and present the results of a usability evaluation (Section 8). First however, we turn to a discussion of related work in this area.

\section{Related Work}

Dalgaard (2001) shifts the attention of scholarly hypertext away from hypertext being merely intertextual relationships between articles to the relationship between text and archive. He points out that from its very inception, hypertext was thought of both at the level of the text and at the level of the network, arguing that in the context of the Web, hypertext has become the paradigmatic rhetorical structure of a global and distributed archive. Accordingly, a scholarly archive is the collection of scholarly texts, and the catalogues and reference works giving access to them. Dalgaard observes that most navigational options are presented as texts (lists of works, authors' names, references, etc)

- this is the hypertextualization of the scholarly archive.

The historical image many people have of the research process that produces these artefacts is that of a lone scientist or small team working in a basement 
laboratory. A similar picture appears for the use of libraries, where researchers ferret away for vital missing bits of information. Levy and Marshall (1995) have examined the early underlying assumptions which have affected digital library development. In their article they challenge these images, especially that digital archives/libraries are used by individuals working alone. They point out that the work carried out by staff (in doing research) and library staff (in providing the service), is one of collaboration, and that digital systems should support formal and informal collaboration and communications.

Similarly, Marchionini and Maurer (1995) point out that 'digital libraries will allow learners of all types to share resources, time and energy, and experience to their mutual benefit'. In their proposed future of digital libraries, sharing resources becomes an important factor supporting teaching, including the ability to share scientific data and other data sets. Many e-science projects have collected a vast amount of data; if the next generation e-scientists are to go beyond the present position they will have to have access to the raw data in their research and training. These early visions are slowly being realised, for example McGrath et al. (1999) have developed a system to locate, navigate and retrieve astronomical data. However, there is still a need for librarians/archivists and users to work together to provide appropriate tools for handling, manipulating and analysing these large data sets (Neville-Manning 2001).

Marchionini and Maurer also suggest that digital libraries should offer greater opportunities for users to deposit information. There are projects beginning to do this, for example the Digital Library for Earth Science Education (DLESE) project allows students to explore geospatial materials and Earth data sets, provides services to create and use materials, and facilitates peer reviewed teaching and learning resources (Marlino et al. 2001). The peer review of collections and peer comment is a significant part of the disseminating process, which adds value to any collection. Weatherley et al. (2002) have proposed a model that will aid reviewers in reviewing complex material or a digital collection. Lyon (2002) sees the digital library, in the context of an information grid, as consisting of a collection of resources for learning and teaching, data repositories for research purposes, or as archives of diverse cultural heritage materials. In her proposed scenario researchers would undertake experiments, deposit raw data, and produce pre-prints using Web services.

We have developed a Web-based environment which we call the Dynamic Review Journal (DRJ) to support such activities in the context of a digital archive: the Virtual European Orthopaedic University (VOEU). Within the DRJ environment, orthopaedic trainees and surgeons can not only collaboratively develop and disseminate the articles which are subsequently managed by the archive, but are also supported in the cycle of activities leading up to the production of these articles - the management of surgical trials, collation 
and analysis of patient data, and organisation of internal project discussions.

\section{VOEU: Motivating Factors}

Recent advances in conventional and computer assisted orthopaedic surgery (CAOS) have resulted in improved clinical outcomes using imaging modalities such as computed tomography and magnetic resonance imaging. Building on this, the computer-based approach extends to other aspects of the surgeons work, including pre-operative planning, surgical simulation and optimisation, intra-operative navigation and robotic systems guidance. With the advent of virtual infrastructures, there is also the potential to manage administrative, research and educational workloads more efficiently and provide greater access to information without the friction associated with traditional infrastructures: the Virtual Orthopaedic European University (VOEU) project was conceived with the goal of building a Virtual University dedicated to education and research in orthopaedics within the European orthopaedic community. However, the availability of such technologies was not the primary driver behind this project - recent trends in orthopaedics and in UK healthcare in general have provided several motivating factors and incentives.

Continuing professional development is an essential part of the healthcare professions: 'life-long learning', to quote the UK Department of Health, 'will provide NHS [National Health Service] staff with the opportunity to continuously update their skills and knowledge to offer the most modern, effective and high quality care to patients'. However, the drive by funding councils towards multi-centred collaborations, the increased mobility of surgeons, the trend towards longitudinal trials, and recent UK government policies to reduce NHS waiting lists and to shorten the training period for new students entering the profession places a greater administrative and management workload on this process whilst also demanding greater availability of up-to-date educational and research materials. There is also increasing pressure from funding councils and charities in the UK to move to a more 'open' community where raw data is published alongside the articles describing which draw conclusions from it, particularly following the recent controversy surrounding MMR vaccinations in children.

\section{VOEU and the Dynamic Review Journal}

The VOEU managed learning environment consisting of hypermedia educational materials (including problem cases and assessment exercises), interactive simulators and communication tools, and is described in more detail in (Conole 
et al. 2003). However, as the project progressed it also became clear from discussions arising from workshops and conferences, and with senior members of the Royal College of Surgeons in the UK that there was also a need for the VOEU to provide support for the research process - the collation and analysis of data in surgical trials and the production of research articles disseminating results to the community. VOEU would thereby provide integrated computer support across the intrinsically coupled research and educational cycles of continuing professional development (research into new techniques and procedures informs updated teaching material for trainees, who will themselves be part of future teams that push the boundaries of knowledge further). An additional Dynamic Review Journal component was therefore conceived which extends the UK arm of the VOEU website (http://voeu.ecs.soton.ac.uk/), adopting the dynamic review (Hazemi and Hailes 1998) approach to data management for orthopaedic research. The processes supported by the DRJ are directly based on a detailed analysis of current practice distilled from discussions, focus groups, and interviews with surgeons in the Wessex (UK) region.

Orthopaedic surgical trials typically run for extended periods (up to 2 years, although there is a trend towards much longer trials emerging e.g. 10 years) and are undertaken (and published) as part of continuing professional development. Upon inception of a hypothesis and the assembling of an investigative team, the first step is to identify a funding source and obtain ethical approval for the trial. The collaborators put together a proposal stating their hypothesis, the exact data they intend to measure and why, and describing the patient group to be studied. This proposal is then sent to a local or national committee for approval - the trial cannot start, nor will funding be received, until approval to carry out the trial is granted.

The next step after approval is to devise a form or spreadsheet (which may be electronic or paper-based) to collect data for the trial. This form is usually based on standard frameworks, such as the American Shoulder \& Elbow Society (ASES) and 'Constant' scoring systems for measuring mobility of the shoulder joint, with some additional variables specific to each particular trial. Each member of the team then receives a copy of the form, and fills in patient data as the trial proceeds. Data collected typically includes pre-operative assessment scores (e.g. range of elbow movement patient can achieve), operational procedure parameters (e.g. type of procedure, anaesthetic), and postoperative assessments (e.g. the patient returns every 6 months over the next 3 years to measure progress). In the case of retrospective investigations, data may also be 'mined' from archived records or personal logbooks, for example examining the case notes of a group of patients who have subsequently developed the same condition in an attempt to identify potential early warning indicators.

After the agreed trial period, the data from individual spreadsheets is col- 
lated and analysed using statistical packages in order to prove/disprove the initial hypothesis and/or to identify potentially interesting patterns or trends in the data. An article describing the results of the trial is collaboratively authored and submitted to a medical journal for peer review. The most prominent journals in the field, the British Medical Journal and the Journal of Bone and Joint Surgery enforce strict structural rules on submissions. For example, the JBJS guidelines state that a structured abstract of no more than 325 words, consisting of five paragraphs with the headings Background, Methods, Results, Conclusions, and Level of Evidence must be provided; the body should consist of the following sections: Introduction, Materials and Methods, Statistical Methods, Results, Discussion - this rigid structure is typical of medical journals. Throughout the trial (and both before and after its 'official' funded duration), collaborators are constantly in contact via telephone, email, or face-to-face meetings.

In supporting this process within the existing VOEU educational framework, the role of the DRJ is therefore two-fold: to aid surgeons in carrying out the research process outlined above, and to support the educational process. Surgical trainees can gain experience in the research process by immersing themselves directly in this environment and can, under supervision from a tutor or team leader, analyse data from existing trials, investigate hypotheses, discuss archived articles, and even prepare and submit their own reports for assessment. This practical (procedural) experience thereby reinforces the declarative (factual) content learnt in the VOEU managed learning environment.

Figure 1 illustrates the major activity spaces in the DRJ and the workflow supported within these spaces.

\subsection{Schema Space}

In general terms, beyond the specific application of the VOEU, the Schema Space is the mechanism by which the Dynamic Review Journal can be configured to the evolving needs of a particular scientific/learning community, through the formal specification of experimentation procedures relevant to that community. This configuration is currently achieved using three different types of schema:

- Data schemas describe the exact nature of the experimental data (for example, specification of variable names, types, and possible values). In the VOEU context, there are a number of data schemas for collecting orthopaedic clinical trial data, including shoulder and hip operation data and post-operative mobility test scores.

- Experiment schemas describe experimental procedures or protocols. For ex- 

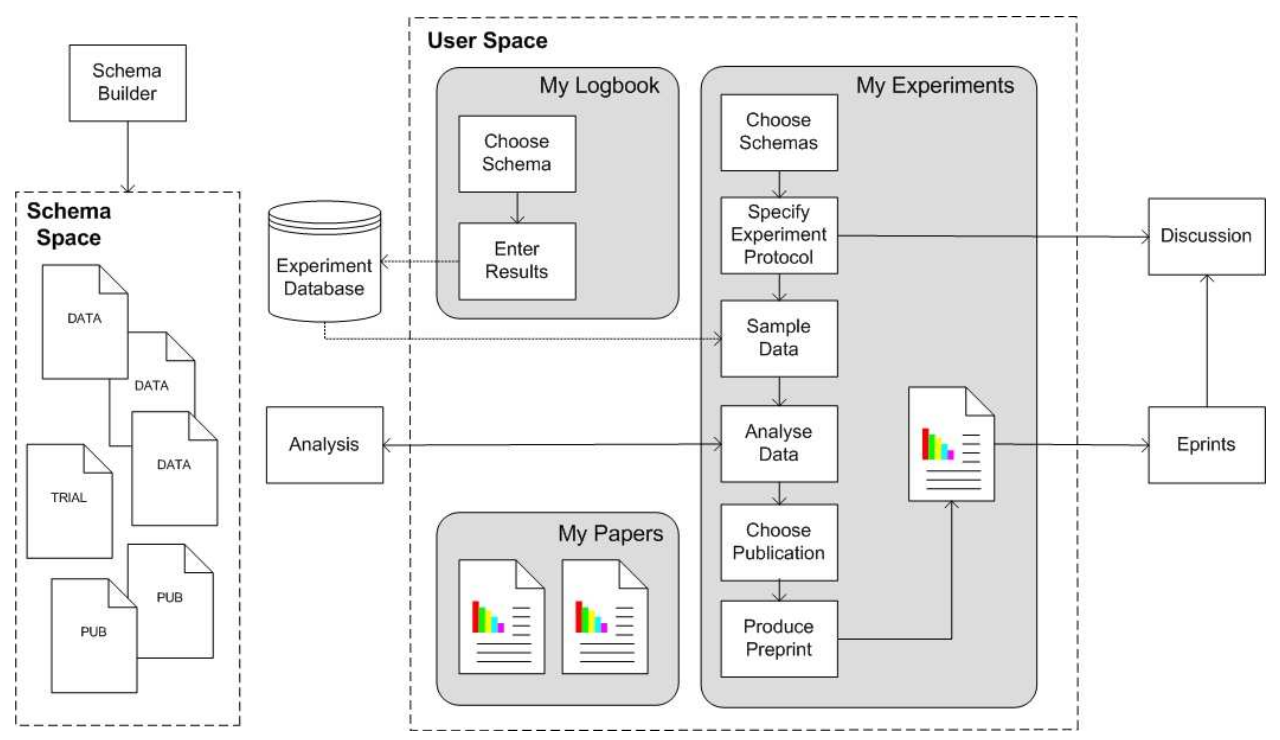

Figure 1. DRJ activity spaces and workflow.

ample, in VOEU a protocol could specify that a surgeon conducting a trial of type X needs to record an experiment description, statement of purpose and an outcome hypothesis. Human-readable guidelines are also included, to help users meet the requirements of the protocol and to help reviewers to ensure that the requirements have been met.

- Publication schemas describe the required format for submitting experimental results to relevant journals or conferences. As with experiment schemas, human-readable guidelines are also included. In the VOEU context, there are currently two publication schemas describing the submission formats for the Journal of Bone \& Joint Surgery (JBJS) and the British Medical Journal (BMJ).

All three types of schema are created using a Template Generation Toolkit, introduced in Section 5.

\subsection{User Space}

The User Space is where users use the schema space to orchestrate practical data entry and collation, experimentation, and dissemination. The User Space is further subdivided into three personalised areas: My Logbook, My Experiments, and My Papers (Figure 2).

My Logbook is an experiment logbook in which experimental results can be entered (in accordance with a selected data schema). Logbook entries are subsequently added to the community database, making data available (anony- 


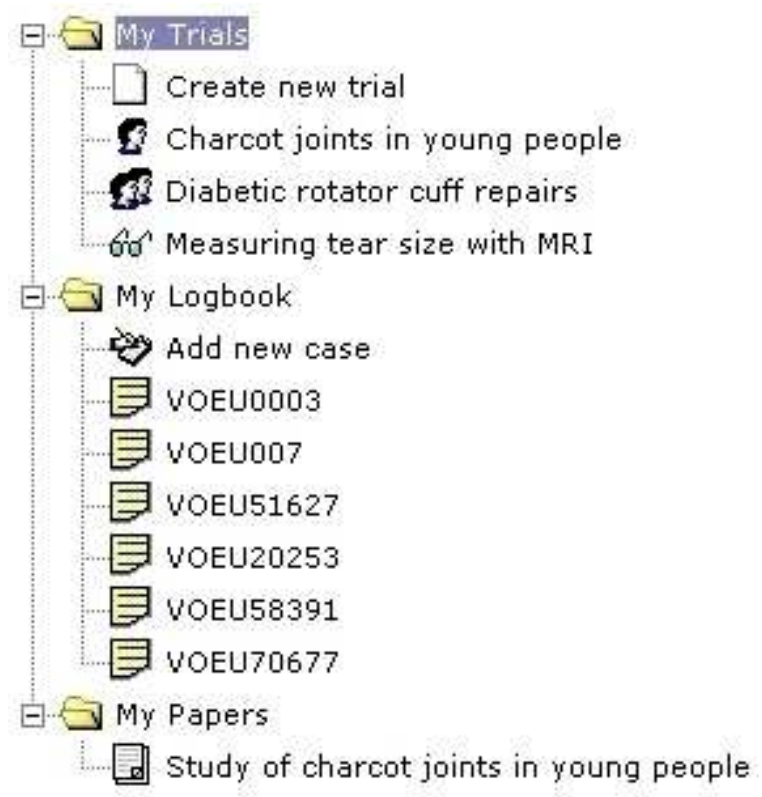

Figure 2. Personalised User Space navigation menu.

mously and with patient consent) to other community members. My Experiments is a workspace for the experiments which the user works on. A user may be involved an experiment in the capacity of lead investigator (initiates experiment and acts as coordinator and contact for duration of experiment), associate investigator (assistant), or reviewer (monitors the progress of the experiment and reviews its outcomes according to agreed guidelines). When a new experiment is initiated, a discussion facility is automatically set up to facilitate and record communication between the users involved (this is also the means by which reviewers can give feed back the practitioners). Figure 1 outlines the work process facilitated by My Experiments:

(i) Define/Choose experiment and data schemas. The lead investigator chooses from the Schema Space the experiment and data schemas which best describe the procedure to be carried out and the data to be collated. If no suitable schemas are available, the lead investigator can create a new schema specifically tailored to the requirements of the experiment. In the case of trainees, a tutor or group leader takes the role of lead investigator.

(ii) Specify experiment protocol. The lead investigator enters the specifics of the experiment protocol (in accordance with the chosen schema), including assigning associate investigators and reviewers to the study. In the case of trainees, the tutor assigns the roles of associate investigators and reviewers to students. 
(iii) Sample data. Investigators create a dataset for the experiment, either by importing their own records from the My Logbook area, or by searching the community database.

(iv) Analyse data. Investigators perform a series of analyses on the dataset, using a distributed analysis service, to test the experiment hypothesis.

(v) Define/Choose publication schema. To initiate the publication cycle, an investigator first chooses the publication schema corresponding to the target conference or journal. Again, if no existing schema meets the requirements of the desired dissemination route, a new schema can be created. In both cases, this produces a 'skeleton' paper. In the case of trainees, the chosen publication schema will usually correspond to an 'assignment report' format.

(vi) Produce outline paper. The investigators proceed to 'flesh out' the paper to produce the beginnings of what will form a pre-print (an article submitted for peer review but not yet accepted). Results from the dataset analyses can be selectively included in the paper.

(vii) Submit to Eprints. The completed paper can be previewed before being automatically submitted to the Eprints digital library component. Subsequent versions of the paper leading to submission, peer review and reprint are managed by the Eprints(Hitchcock et al. 2000) server. Investigators continue to discuss the development of the paper in the discussion forum.

It should be noted that the work-flow is not enforced as a linear progression from experiment protocol to pre-print; investigators can make changes to the experiment protocol as the experiment progresses (for example, bringing a new associate investigator on board, inviting a new student to join the exercise), return to the dataset at any point to add/remove experimental results or perform more analyses, and produce many different pre-prints describing different aspects of the experiment.

Finally, My Papers provides a simple shortcut allowing the user to quickly access all the papers and reports produced by the various experiments and exercises worked on.

\section{$5 \quad$ Evolving to Changing Needs of Users}

In order for an approach such as the DRJ to succeed, the digital library environment needs to be able to evolve to meet the changing needs of its user community - it is unreasonable to expect the developers of such a system to predict and encode every possible experimental procedure, every type of data that can be collated and analysed, and every possible dissemination route that users will follow to publish their results to the wider community. The Schema 
Space is therefore the fundamental aspect of the design of the DRJ, providing the mechanism by which the DRJ can be configured to the requirements of a community (in the case of the VOEU, the community of orthopaedic surgeons and trainees within the EU), a particular group of users, or even an individual. The schemas in the Schema Space provide an interface between the data stored by the system and the users' view of that data for example, in the case of the data schema, the type and format of sets of data variables are specified and subsequently used to dynamically generate user interfaces for entering, viewing, and performing statistical analyses.

Until recently, this mechanism was only available to those users with the appropriate technical nous to formalise their data requirements using the schema grammar some of our most recent work has therefore focused on building a toolkit to enable the wider (non-expert) VOEU community to configure the DRJ to the particular requirements of their experimental activities. The first barrier to achieving this goal was the use of the term 'schema' - for surgeons, this is an alien term within their day-to-day work experience, so the more familiar term 'template' was chosen in its place. This change of name from schema to template also emphasised the evolutionary nature of most schemas. Rather than start with a blank schema and add new items, the majority of new schemas would be modifications of existing ones. Therefore an existing schema could form a template around which new ones could be created. The DRJ's schema-building component thus became known as the Template Generation Toolkit.

\subsection{Template Generation Toolkit}

The Template Generation Toolkit (TGT) enables non-expert users to quickly and easily add new schema specifications, or 'templates' to the DRJ Schema Space, in accordance with their specific requirements. The TGT has been developed in the form of a pedagogical toolkit - such toolkits guide users through the process of articulating their information needs to produce an information plan for a particular task (Conole et al. 2003). Pedagogical toolkits lie between restrictive wizards which force a user through a particular route (for example, a printer installation wizard) and more open frameworks which provide unrestricted access to a different parts of a creation process. The TGT therefore, is designed to provide a structured way of creating new schemas.

In the case of data schemas, variables can be grouped together into sets based on the type of data they record. For example, a patient data set could include age, weight, height, and ethnic origin, while a data set for recording the range of movement in Perthes disease sufferers could include range and degree of motion in various directions, pain experienced, and muscle response. New data schemas can then be built using sets defined by existing schemas and 


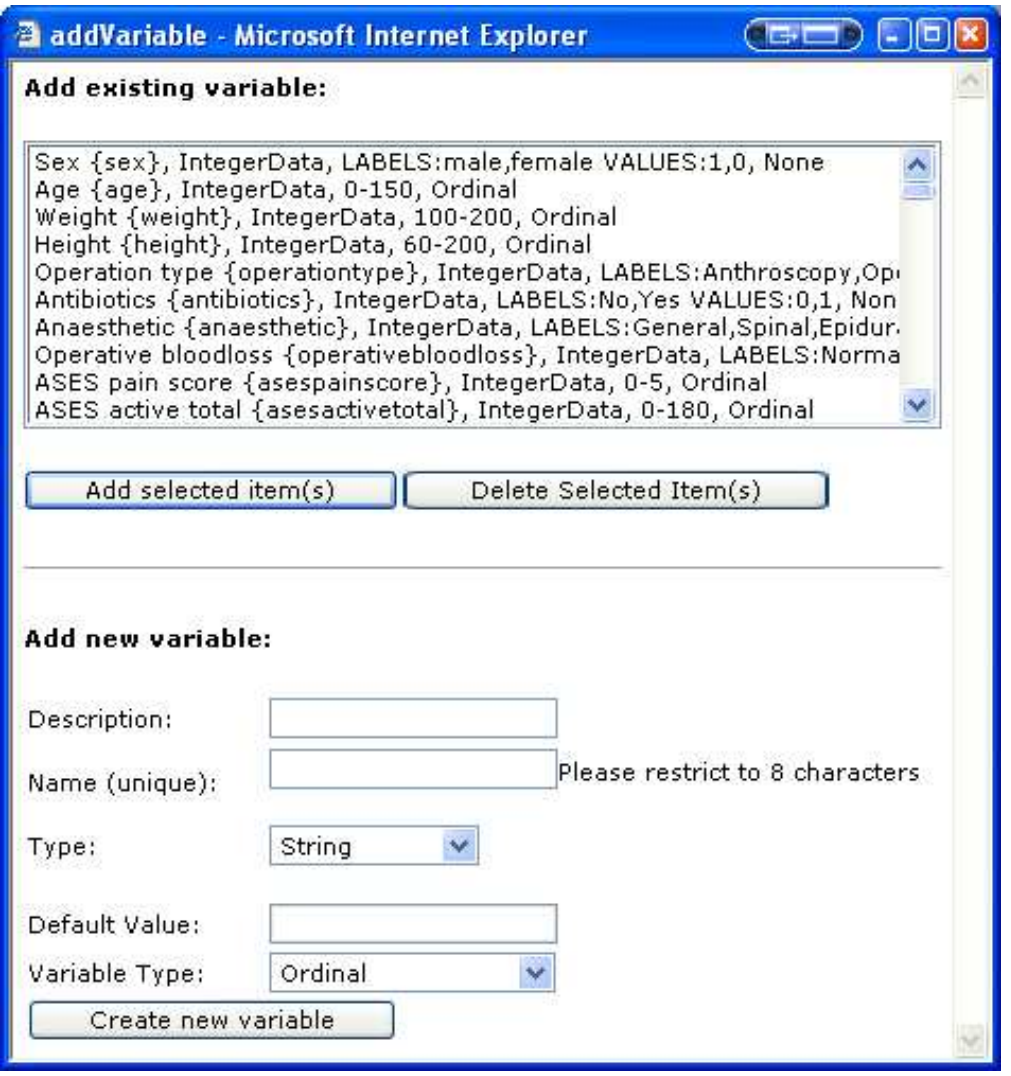

Figure 3. Adding a new variable to a schema in the Template Generation Toolkit.

'fine-tuned' by adding, deleting, or modifying individual variables (Figure 3). Slots for image data such as photographs, x-rays, and digitised sketches can also be specified within a data schema.

Once a data schema has been created using the Template Generation Toolkit, it is immediately available to members of the VOEU community to use to input new case data. When a suitable number of cases based on the new schema have subsequently been entered into the VOEU database, the dataset can be searched and analysed.

\subsection{Issues Raised}

The development work on the Template Generation Toolkit has raised some issues within the context of the VOEU community, which have wider implications that must be borne in mind as we move towards our goal of more widespread adoption of a Dynamic Review Journal-based infrastructure sup- 
porting the experimental work of other on-line scientific communities (see Section 6).

One issue that was faced concerned the lack of presentation information defined within the data schema. Without this information, the user interfaces generated from the data schema (including data entry forms, data viewing tables, search forms, and data analysis forms) are simplistic and simply list each variable in the order that they are defined in the schema. While this was sufficient in most cases, in specific cases we were asked by surgeons to adjust the appearance of the forms so that they mirrored their paper-based counterparts. This issue potentially hints at a much larger one - that in largescale trials involving participants from multiple institutes throughout the EU there is a much greater need for customised presentation of information then would be required in the case of a smaller trial whose participants are all part of the same institute. Issues such as cultural, linguistic and political differences all need to be properly addressed. VOEU currently holds 'user profile' information for each registered user of the system, which has provided a useful starting point for our work in this area to provide personalised interfaces in different languages or presentation styles.

Another issue facing multi-national trials is the potential difference in local policies between participating institutions; a VOEU-specific example is that of different policies used in different regions for assessing a patient's pre- and post-operative motor facilities - surgeons in the US use the American Shoulder and Elbow Society (ASES) system, whereas in the EU the CONS system is used. The former measures shoulder movement by degrees, the latter measures the same movement on a scale of one to five. A mechanism to enable the DRJ to map between the different types of assessment could be defined in this instance, allowing scores from both types of system to be compared and analysed side by side; however, there may be some cases in which a measurement in one system has no equivalent in the system to which it is being compared.

\section{Deploying the DRJ in other Online Communities}

Although originally conceived as part of the VOEU, the Dynamic Review Journal has been developed as a generic framework with the aim of being adopted as an infrastructure to support the experimental work of other online scientific communities. Figure 4 illustrates the framework concept, in the context of an e-science community Web site/archive. Implemented as a toolkit of Microsoft.NET components, the DRJ can be integrated with existing sites. Although the framework itself provides data storage and management capabilities, facilities have also been provided to help communities integrate the DRJ with existing data repositories. Distributed Eprints, discussion, and analysis 


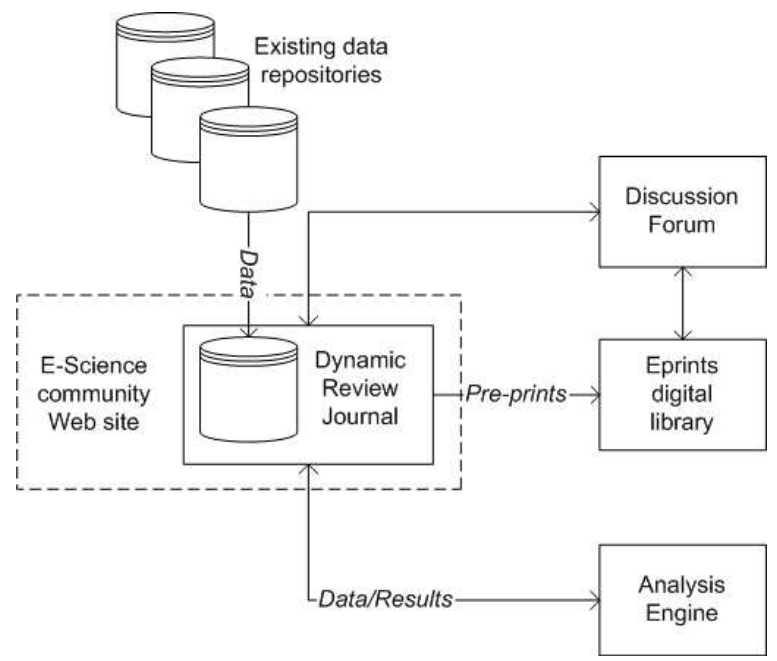

Figure 4. Conceptual overview of the DRJ

services provide integrated support for document management, communication, and experimentation respectively.

However, we acknowledge that the orthopaedics-specific workflow supported by the DRJ may prove too restrictive for other communities in which research practices are more explorative. For example, within the field of orthopaedics there are very restrictive requirements and formats for specifying data and publishing papers which fit very well with the current template-driven model, but in other fields publishers' requirements may more open-ended. We are therefore looking at ways in which a less rigid workflow processes could be supported. Basic Support for Cooperative Work (BSCW) (Appelt 1999), for example, provides Web-based shared workspaces allowing collaborating users to collect and structure any kind of information (including, but not limited to, documents, images, spreadsheets, software, and URL links to other Web pages or FTP sites) in order to achieve their goals of collaboration. Users primarily access workspaces through a normal browser (no additional software required), although a separate BSCW events server feeds a downloadable monitor applet with events about the presence and activities of other users in the workspace, allowing users to better coordinate their work.

In line with the framework illustrated in Figure 4, a distributed BSCW server component could manage shared workspaces for each experiment, enabling the investigators to collaborate in an unstructured (explorative) manner at each stage of the experiment whilst still being driven by the underlying DRJ workflow process. As an example, at the publication stage the experiment metadata and selected results could be "exported" to the shared workspace where the investigators mould it into a preprint outside of the rigid DRJ 


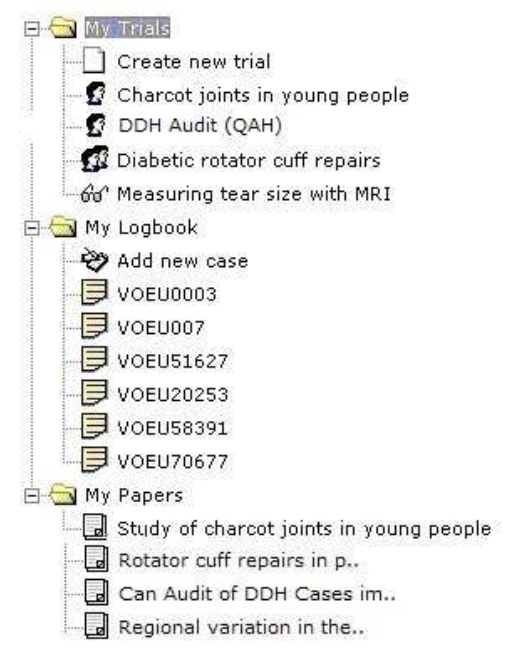

Figure 5. Simon's DRJ user space.

template model, before making it available to the community via the Eprints server.

\section{$7 \quad$ Case Study}

To illustrate the DRJ in use, this section provides an account of a user, Simon Grange (co-author of this paper), performing a surgical trial which investigates Perthes disease, a hip disorder found in children.

Figure 5 shows Simon's view of the DRJ user space (note that this navigation menu is always available, but excluded from some figures for clarity. Simon is currently working on four trials, undertaking a different role in each (note that roles are depicted using icons next to each trial). Simon is the lead investigator in the 'charcot joints' and 'DDH Audit' trials (note that DDH means developmental dysplasia of the hip, a condition where a child's hip joint does not grow normally), an associate investigator in the 'rotator cuff' trial, and reviews the 'tear size' trial. Simon has also entered several records in his personal logbook (patient details, operative procedures, and assessment results), and so far has produced four papers.

To initiate the DDH trial (having previously received approval from an ethics committee), Simon first selected the orthopaedic clinical trial protocol from the available experiment schemas. The DRJ then uses this schema to generate a number of data entry forms in which Simon enters specifics of the trial (Figure 6).

Guidelines for completing these forms are presented as 'stretchtext 


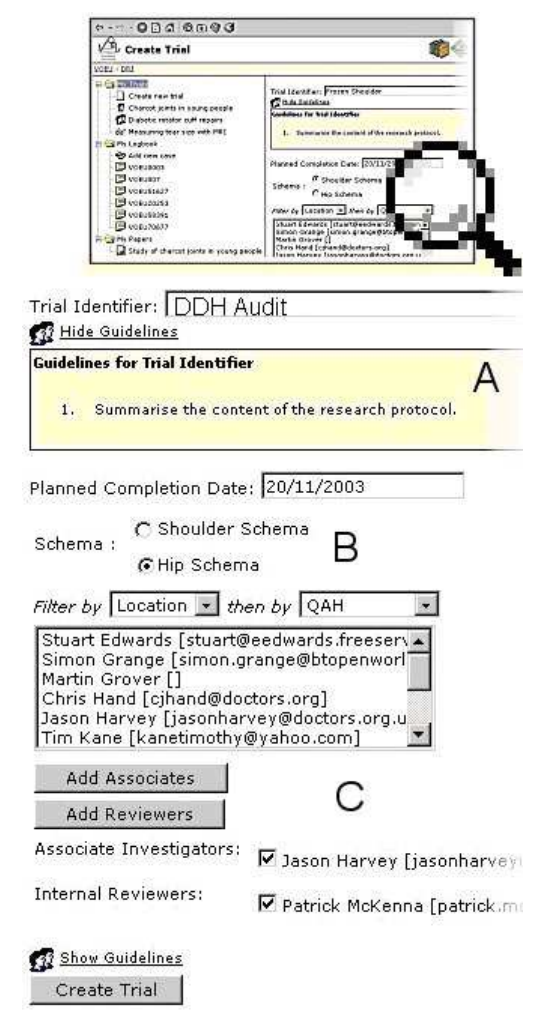

Figure 6. Entering trial protocol information.

links' (Nelson 1987) which can be viewed/hidden as required (Figure 6A). Simon also specifies that the hip data schema will be used in the trial (Figure $6 \mathrm{~B}$ ) and lists the associative investigators and reviewers who will assist him on the trial (Figure 6C). If no existing schema had satisfied the data requirements of this trial, Simon could use the Template Generation Toolkit to define a new schema based on the existing hip schema. When created, the new trial appeared in Simon's DRJ user space (as we saw in Figure 5), and also in the user spaces of his named associate investigators and reviewers. The experiment protocol may subsequently be updated by any of the investigators, perhaps as a result of critical comment from the reviewer.

As the trial progresses, Simon and his colleagues use the My Logbook area to add new case data to the trial (currently, Simon keeps a shadow log on paper in line with NHS policy). Logbook data subsequently becomes available to the rest of the community (provided that the surgeon has marked it as public) through the patient database; therefore, Simon can also add any existing data from past hip studies to the trial data set by searching the VOEU database of patient records. Since the hip schema has already specified for this trial, 


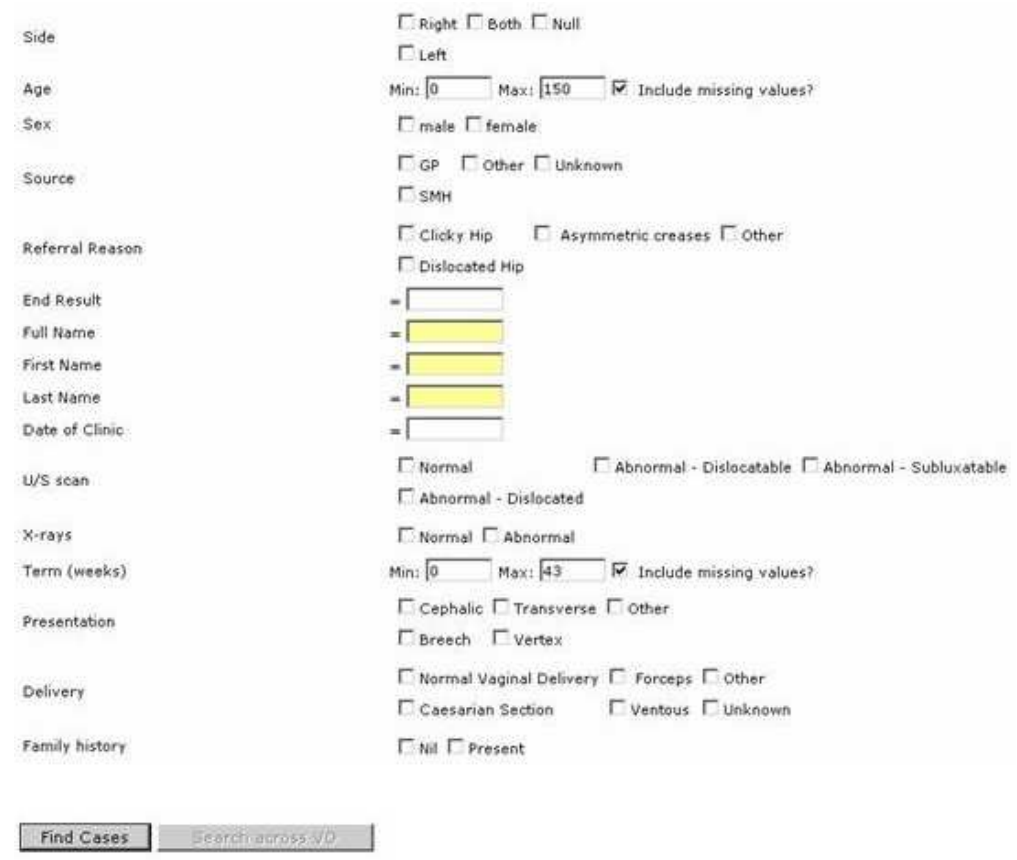

Figure 7. Searching archived patient records.

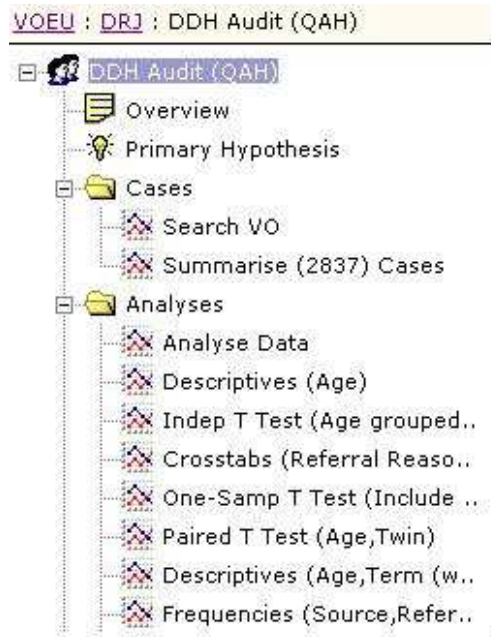

Figure 8. Trial navigation submenu. 


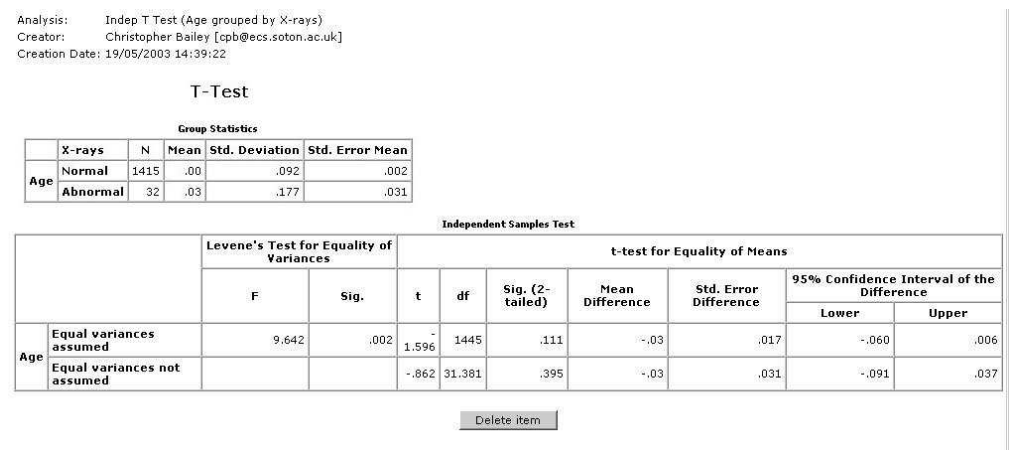

Figure 9. Viewing analysis results.

only those cases matching this schema will be searched. The DRJ uses the hip schema to generate a search form, so that Simon can search for appropriate cases (Figure 7). Using both logbook entry and the patient database, Simon and his associates have subsequently added 2837 different hip cases to the DDH trial (see trial navigation menu in Figure 8), which can be tabulated (summarised) for visual comparison. The bulk of the data entry work is currently undertaken by Simon's junior colleague. This also provides the 'audit trail' back to the selected cases. To perform analyses on this data set, Simon and his associates choose from statistical methods offered by a distributed Analysis Engine. Using the hip data schema and metadata from the engine, the DRJ is able to generate an configuration form for each statistical method, which is used to fine tune the analysis (specify test variables, groupings, etc.). The Analysis Engine queues the requested analysis and notifies the DRJ when results are available. These results then appear in Simon's DRJ user space, and can be viewed (Figure 9).

Having obtained some significant results from the statistical analyses, Simon then decides to create a pre-print in order to disseminate the results to the wider orthopaedic community. When Simon selects the JBJS publication schema, the DRJ generates a pre-print template using the information Simon entered in the trial protocol (e.g. the hypothesis entered in the protocol is automatically entered into the Introduction section, as the JBJS guidelines instruct). Simon fleshes out this template, following the JBJS guidelines provided (Figure 10), and specifies which analysis results should be included in the pre-print. After previewing the pre-print, Simon submits it: behind the scenes the DRJ submits the pre-print and its associated metadata to the community Eprints server (where it subsequently becomes available to the VOEU community), and makes the paper available in the user space of Simon and associates.

Subsequent versions of the pre-print leading to submission, peer review, and 


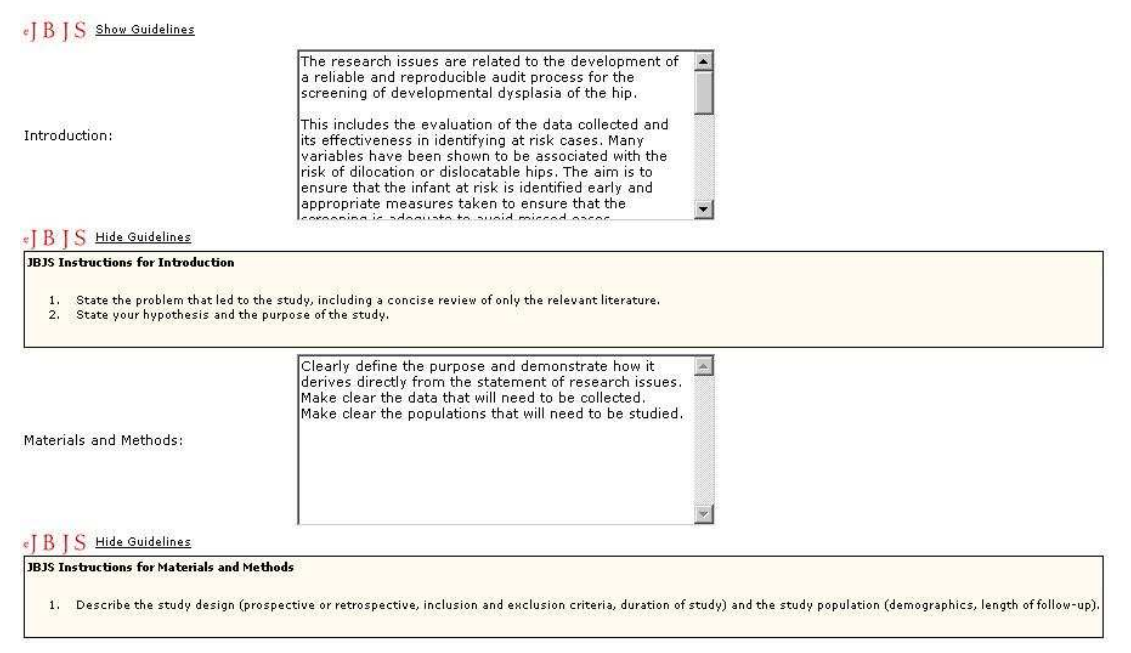

Figure 10. Preparing pre-print.

reprint are managed by the Eprints server. Simon and associates continue to discuss the development of the paper in the discussion forum. If the article is subsequently accepted by the target journal, the record is upgraded to a 're-print' - community members can therefore easily distinguish work that has been accepted by peer review.

\section{Evaluation}

The VOEU project has recently carried out a broad usability evaluation of the range of services offered by the UK Web site. Although this evaluation focused on capturing user's general responses to the overall VOEU 'experience' rather than any specific features of the DRJ, the results nevertheless provide some useful insights into the applicability and utility of the DRJ, as we look ahead to a more in-depth user evaluation of the DRJ's experimentation, analysis, discussion, and publication tools.

The evaluation was carried out by 18 orthopaedic surgeons with a mean age of 30.2 (SD 4.6), and a mean of 5.1 years surgical experience (SD 4.3). The majority of the participants responded to background questions in a way that indicated that they were 'tech-savvy' - they understood the benefits of electronic access to information, used the Web regularly at home and work, and preferred the electronic medium over traditional mediums. Even those who were less tech-savvy acceded the benefits of electronic access - only one participant, a self-confessed 'techno-phobe', maintained that paper-based materials were the easiest and preferred working medium.

Each participant followed a tour through the VOEU Web site, with each area 
being demonstrated before allowing the participant to familiarise themselves with its function through 'hands-on' experimentation. Participants were also invited to carry out simple tasks, such as finding information in the VOEU digital library, and taking part in an interactive surgical simulation. In the case of the DRJ, each participant was given the opportunity to run through the entire process of setting up a clinical trial, carrying out data collection and analysis, and producing a targeted pre-print. Each participant then completed a questionnaire designed to capture their responses to a number of different aspects of their VOEU 'experience', based on the Software Usability Measurement Inventory (SUMI) (Kirakowski and Corbett 1993), including extensions proposed by Crowder et al. (2003) for evaluating hypermedia systems. SUMI enabled us to measure several different aspect of the VOEU experience:

- Aidability - The degree to which the VOEU site assists the user to resolve a situation.

- Command - The extent to which the user feels that they are in control.

- Comprehension - The degree to which the user understood the interaction with the VOEU site.

- Effectiveness - The degree to which the user feels that they can complete the task within the VOEU site.

- Impression - The user's feelings or emotions when using the VOEU site.

- Learnability - The degree to which the user feels that the VOEU site is easy to become familiar with.

- Navigability - The degree to which the user can move around the VOEU site.

The results of the evaluation are shown in Figure 11, where a mean response value of 5.0 indicates an entirely positive result, and a mean response of 1.0 indicates an entirely negative result. Initial indications from this trial therefore show a positive response to all aspects of the VOEU usability experience. The greatest positive responses were to the statements 'I was able to move around the information in VOEU easily' (navigability), 'learning to use the system was easy' (learnability), 'I felt at ease trying different ways to get to the information I needed' (learnability), 'the system help files provided enough information to use the system' (aidability), 'VOEU could be of use to me in my job' (effectiveness), and 'using VOEU allows me to accomplish tasks more quickly' (effectiveness). Areas which proved more controversial included 'I often become lost/disoriented when using VOEU', 'it was difficult to learn more than the basic functions of the VOEU system', and 'the system was awkward to use if I wanted to do anything out of the ordinary'.

In order to better focus future developments of the DRJ and other VOEU services, participants were also asked in a separate questionnaire to rank a number of proposed VOEU extensions in order of relative importance to their 


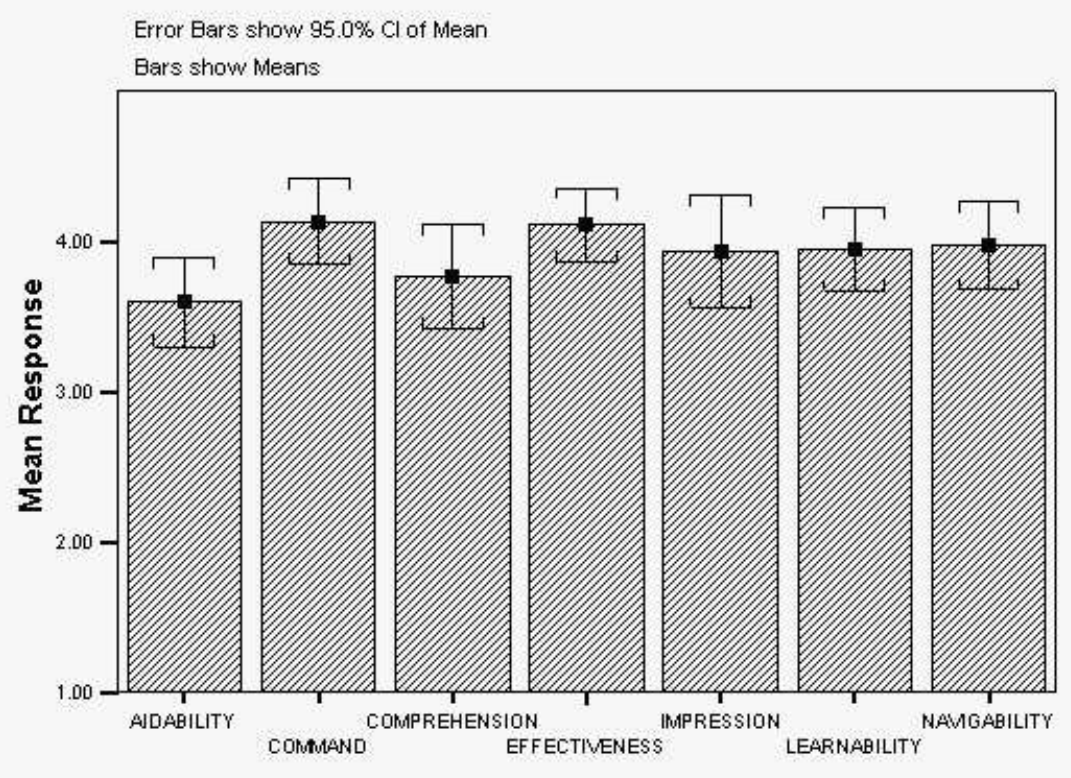

Figure 11. Results of the VOEU usability trial, showing average participant responses for different areas of their VOEU 'experience'.

Table 1. Proposals for extensions to the VOEU/DRJ environment, as ranked by participants in order of relevance.

\begin{tabular}{cl}
\hline Rank & Proposed Extension \\
\hline $1(1)$ & $\begin{array}{l}\text { Automatic uploading of trial data to other records, such as } \\
\text { the BOA logbook JCHST, GMC revalidation. }\end{array}$ \\
$2(3)$ & $\begin{array}{l}\text { Pervasive access from handheld devices (e.g. trial data en- } \\
\text { try from PDA). }\end{array}$ \\
$3(4)$ & $\begin{array}{l}\text { Heuristic support for users unfamiliar with statistics man- } \\
\text { agement. }\end{array}$ \\
$5(5)$ & $\begin{array}{l}\text { Journal submission templates for all leading journals. } \\
5(7)\end{array} \begin{array}{l}\text { Enhanced trial data entry. } \\
6(9)\end{array}$ \\
\hline
\end{tabular}

day-to-day work. Table 1 lists the ranked proposals which are specific to the DRJ (with overall ranking shown in parentheses).

\section{Conclusions}

In this paper we have taken the position proposed by Dalgaard that scholarly hypertext is not merely the intertextual relationships between papers but 
the relationships between text and the wider archive (Dalgaard 2001). In our view a digital archive is more than just a collection of digital copies of documents - we argue for a broadening of this view, out from the document collections themselves, to the intertextual process of creating, collecting and deploying them. Parts of the scientific community (and the computing community) are currently obsessed by the idea of the Grid the use of computers to improve scientific experimentation, analysis, and collaboration. However, we argue that publishing, dissemination, research, and learning are equally important (perhaps more important) parts of the scientific cycle and should not be left to unaided manual effort. This amounts to the hypertextualization of the scientific process.

We have presented our contribution to this ongoing effort, the Dynamic Review Journal, and described its motivation, integration and use within a Virtual University environment, as an example of a system which deliberately crosses the barriers between these areas (experimentation, analysis, publishing, dissemination, discussion, and education). In providing support for the broader range of scientific activities, it is our hope that scientists can be made more effective in their work. We have received further funding to expand the DRJ into a Virtual Research Environment (VRE), to be called the Collaborative Orthopaedic Research Environment (CORE), using a Web-services based distributed architecture.

\section{Acknowledgements}

Part of this work was funded by The European Commission under the Virtual Orthopaedic European University project, IST-1999-13079, Information Society Technology Program.

\section{REFERENCES}

Appelt, W., WWW based collaboration with the BSCW system. In: Proceedings of the 26th Annual Conference on Current Trends in Theory and Practice of Informatics, Milovy, Czech Republic, pp. 66-78, 1999.

Carr, L., Miles-Board, T., Wills, G., Power, G., Bailey, C., Hall, W. and Grange, S., Extending the role of the digital library: Computer support for creating articles. In: Proceedings of the Fifteenth ACM Conference on Hypertext and Hypermedia, Santa Cruz, California, USA, pp. 12-21, August 2004.

Conole, G., Wills, G., Carr, L., Vadcard, L., Hall, W. and Grange, S., Building a virtual university for orthopaedics. In: Proceedings of the World Confer- 
ence on Educational Multimedia, Hypermedia and Telecommunications, Honolulu, Hawaii, pp. 22-27, 2003.

Crowder, R., Wills, G. and Hall, W., Evaluation of a hypermedia maintenance support application. International Journal Computers in Industry, 2003, $\mathbf{5 1}(3), 327-344$.

Dalgaard, R., Hypertext and the scholarly archive: Intertext, paratexts and metatext at work. In: Proceedings of the Twelfth ACM Conference on Hypertext and Hypermedia, Arhus, Denmark, pp. 175-184, August 2001.

Frey, J., De Roure, D. and Carr, L.A., Publication At Source: Scientific Communication from a Publication Web to a Data Grid. In: Proceedings of the EuroWeb 2002, Oxford, UK, December 2002. Available online at http://ewic.bcs.org/conferences/2002/euroweb/session3/paper3.htm

Ginsparg, P., Winners and Losers in the Global Research Village. In: Proceedings of the 1996 UNESCO Conference, Paris, February 1996. Available online at http://xxx.lanl.gov/blurb/pg96unesco.html

Harnad, S., Electronic Scholarly Publication: Quo Vadis? Serials Review, 1995, 21(1), 70-72.

Hazemi, R. and Hailes, S., Reinventing the academy. In: The Digital University, Wilbur, S., Hazemi, R. and Hailes, S. (Eds), pp. 7-24, 1998, (London: Springer).

Hitchcock, S., Carr, L. and Hall, W., A Survey of STM Online Journals 199095: the Calm Before the Storm. In: Directory of Electronic Journals, Newsletters and Academic Discussion Lists (6th edn), Mogge, D. (Ed), pp. 7-32, 1996, (Washington DC: Association of Research Libraries). Available online at http://journals.ecs.soton.ac.uk/survey/survey.html.

Hitchcock, S., Carr, L., Jiao, Z., Bergmark, D., Hall, W., Lagoze, C. and Harnad, S., Developing services for open eprint archives: globalisation, integration and the impact of links. In: Proceedings of the Fifth ACM Conference on Digital Libraries, San Antonio, Texas, USA, pp. 143-151, 2000.

Kirakowski, J. and Corbett, M., SUMI: The Software Usability Measurement Inventory. British Journal of Educational Technology, 1993, 24(3), 210212.

Levy, D.M. and Marshall, C.C., Going digital: a look at assumptions underlying digital libraries. Communications of the ACM, 1995, 38(4), 77-84.

Lucier, R.E., Knowledge management: Refining roles in scientific communication EDUCOM Review, Fall 1990, 21-26.

Lyon, L., Emerging information architectures for distributed digital libraries. In: Proceedings of the Digital Library - IT Opportunities and Challenges in the New Millennium Conference, Beijing, China, July 2002.

Marchionini, G. and Maurer, H., The roles of digital libraries in teaching and learning. Communications of the ACM, 1995, 38(4), 67-75. 
Marlino, M., Sumner, T., Fulker, D., Manduca, C. and Mogk, D., The digital library for earth system education: building community, building the library. Communications of the ACM, 2001, 44(5), 80-81.

McGrath, R.E., Futrelle, J., Plante, R. and Guillaume, D., Digital library technology for locating and accessing scientific data. In: Proceedings of the fourth ACM conference on Digital Libraries, Berkley, California, pp. 188-194, August 1999.

Nelson, T.H., Computer Lib/Dream Machines, 1987, Redmond, WA: Tempus Books.

Nevill-Manning, C., The biological digital library. Communications of the $A C M, 2001,44(5), 41-42$.

Weatherley, J., Sumner, T., Khoo, M., Wright, M. and Hoffmann, M., Partnership reviewing: a cooperative approach for peer review of complex educational resources. In: Proceedings of the Joint Conference on Digital Libraries, Portland, Oregon, USA, pp. 106-114, 2002. 